\title{
Reducing diarrhoea deaths in South Africa: costs and effects of scaling up essential interventions to prevent and treat diarrhoea in under-five children
}

Lumbwe Chola $^{\dagger}$, Julia Michalow ${ }^{\dagger}$, Aviva Tugendhaft and Karen Hofman $^{*}$

\begin{abstract}
Background: Diarrhoea is one of the leading causes of morbidity and mortality in South African children, accounting for approximately $20 \%$ of under-five deaths. Though progress has been made in scaling up multiple interventions to reduce diarrhoea in the last decade, challenges still remain. In this paper, we model the cost and impact of scaling up 13 interventions to prevent and treat childhood diarrhoea in South Africa.

Methods: Modelling was done using the Lives Saved Tool (LiST). Using 2014 as the baseline, intervention coverage was increased from 2015 until 2030. Three scale up scenarios were compared: by 2030,1) coverage of all interventions increased by ten percentage points; 2 ) intervention coverage increased by 20 percentage points; 3) and intervention coverage increased to $99 \%$.

Results: The model estimates 13 million diarrhoea cases at baseline. Scaling up intervention coverage averted between 3 million and 5.3 million diarrhoea cases. In 2030, diarrhoeal deaths are expected to reduce from an estimated 5,500 in 2014 to 2,800 in scenario one, 1,400 in scenario two and 100 in scenario three. The additional cost of implementing all 13 interventions will range from US\$510 million (US\$9 per capita) to US\$960 million (US\$18 per capita), of which the health system costs range between US\$40 million (less than US\$1 per capita) and US\$170 million (US\$3 per capita).

Conclusion: Scaling up 13 essential interventions could have a substantial impact on reducing diarrhoeal deaths in South African children, which would contribute toward reducing child mortality in the post-MDG era. Preventive measures are key and the government should focus on improving water, sanitation and hygiene. The investments required to achieve these results seem feasible considering current health expenditure.
\end{abstract}

Keywords: Modelling, Diarrhoea, Child health, Cost analysis, South Africa

\section{Background}

Globally, one in 10 deaths in children under the age of five years results from diarrhoea, with the majority occurring in sub-Saharan Africa and South East Asia [1]. Diarrhoea is one of the leading causes of morbidity and mortality in under-five children in South Africa, however the true burden of childhood diarrhoea is not accurately

\footnotetext{
* Correspondence: karen.hofman@wits.ac.za

${ }^{\dagger}$ Equal contributors

Priority Cost-Effective Lessons for Systems Strengthening South Africa (PRICELESS SA) - Medical Research Council/Wits Rural Public Health and Health Transition Research Unit (Agincourt), School of Public Health, Faculty of Health Sciences, University of the Witwatersrand, Johannesburg, South Africa
}

known. Official data from Statistics South Africa estimate that diarrhoea accounts for approximately $20 \%$ of underfive deaths [2], but other sources estimate the burden between 8\% [1] and 13\% [3]. The 2010 General Household Survey (GHS), a nationally representative inquiry into the livelihood of South Africans, showed that there were over 60,000 cases of childhood diarrhoea per month and approximately 9,000 child diarrhoeal deaths in the same year [2].

Diarrhoea is closely linked to socio-economic status and has the most adverse effects in South Africa's impoverished communities [2,4]. South African children living 
in poverty are approximately ten times more likely to die from diarrhoea than their more privileged counterparts [2]. Poor nutritional status, poor environmental conditions, and illnesses such as HIV/AIDS make children more susceptible to severe diarrhoea and dehydration [4]. Episodes of persistent diarrhoea also worsen a child's condition and nutritional status due to decreased food intake and nutrient absorption [4]. In HIV-infected children, persistent diarrhoea is associated with an 11-fold increase in mortality [5]. More than 50\% of South African children who died in 2012 had evidence of HIV infection or exposure, while $60 \%$ were undernourished [6].

UNICEF and WHO have stressed the importance of well-known interventions for reducing the global burden of childhood diarrhoea $[4,7]$. Interventions for diarrhoea prevention include vaccinations against rotavirus, cholera, typhoid and measles; micronutrient supplementation for zinc and vitamin A; prevention and treatment of comorbidities, such as HIV; exclusive breastfeeding promotion and support; adequate nutrition for mothers and children; and interventions for the provision of water, sanitation and hygiene (WASH). Diarrhoea should be treated with oral rehydration solution (ORS), zinc, continued feeding, antibiotics for dysentery, as well as improved care seeking behaviour and improved case management.

Progress is being made towards implementing these interventions. In 2009, South Africa became the only country in sub-Saharan Africa to include the rotavirus vaccine in routine child immunizations. The vaccine, which has been shown to be effective in preventing severe rotavirus diarrhoea [8], has achieved moderate coverage in South Africa (64\%) [9]. The government revised its breastfeeding policy in 2011 to actively promote exclusive breastfeeding and phase out the distribution of free infant formula to babies born to HIV-positive mothers. South Africa has also achieved the water and sanitation targets for Millennium Development Goal (MDG) 7; over 90\% of South Africans have access to a clean public water source and over $70 \%$ utilize a latrine or toilet [10]. However, despite achieving these goals, approximately six million households $(46 \%)$ do not have access to piped water in their homes and 1.4 million households (11\%) still lack access to sanitation services [11]. Furthermore, the sanitation services in over 3.8 million households (26\%) in formal areas do not meet the required standards due to infrastructure deterioration [11]. Coverage remains low for many of the other recommended interventions, such as hand washing with soap and ORS. Although these health promotion interventions are affordable, there are significant challenges to increasing adoption.

The disparities in access to water and sanitation services, and the poor coverage of essential interventions contribute to the ongoing high prevalence of diarrhoea in the country. This analysis evaluates the potential impact of scaling up coverage of the recommended interventions on under-five diarrhoeal mortality in South Africa between 2014 and 2030. The potential number of lives that could be saved and the resources required for intervention scale up are assessed in order to aid priority setting and budgeting. The results of this analysis could aid South Africa's plans to reduce child mortality in the post-2015 era.

\section{Methods}

\section{Lives Saved Tool (LiST)}

Modelling was done using the Lives Saved Tool (LiST), a module in the Spectrum software [12]. Version 5.07 was used (downloaded 28/11/2014). LiST is a deterministic mathematical model that compares the effect of various interventions on population level risk factors, as well as stillbirths and maternal, newborn and child deaths $[13,14]$. Included in the model are more than 60 interventions that have an impact on cause-specific mortality. An intervention can have an impact on single or multiple causes of death and risk factors. The outcome measures (risk factors and cause-specific mortality) change based on the level of coverage of the interventions included in the model. Increasing the level of coverage of one or more interventions can thus lead to a reduction in associated risk factors or causespecific mortality. LiST therefore enables a user to assess the simultaneous impact of interventions on health outcomes. Intervention impact on mortality can be direct or indirect (through the reduction of risk factors). The direct impact of each of these interventions is modelled by multiplying its effectiveness estimate with the level of coverage, assuming all other interventions are kept constant. For example, an intervention with an effect estimate of $30 \%$ can avert $30 \%$ of the associated cause-specific deaths if coverage for that intervention is $100 \%$.

When LiST analyses multiple interventions, each intervention is applied to the residual deaths from the previous intervention. This prevents double counting the number of lives saved. The model starts by applying the preventive interventions in succession, followed by the treatment interventions on remaining deaths. The total number of deaths prevented is therefore not attributable to specific interventions but rather the full intervention package [15].

LiST includes 14 interventions for the prevention and treatment of diarrhoea. Walker and Walker (2014) describe the interactions between these interventions and the modelling methods used in LiST [15]. There are 12 interventions in LiST that have a direct impact on diarrhoeal mortality. Eight of these are preventive 
interventions: rotavirus vaccine; vitamin A supplementation; zinc supplementation; and the water, sanitation and hygiene (WASH) programmes that include a water connection in the home or improved water source, improved sanitation hand washing with soap and hygienic disposal of children's stools. Interventions for breastfeeding promotion, severe wasting and moderate acute malnutrition have an indirect impact on diarrhoeal mortality. The impact of breastfeeding can be modelled either as a risk factor that changes when breastfeeding promotion shifts breastfeeding rates, or as a direct risk factor for death due to the lack of appropriate breastfeeding. (In our analysis, we ramped up breastfeeding according to WHO guidelines, which recommend six months of exclusive breastfeeding and appropriate complementary feeding up to two years). LiST also includes three interventions for treating diarrhoea: zinc treatment, ORS and antibiotics for dysentery. Figure 1 (adapted to reflect the interventions addressed in our analysis) provides an overview of the intervention interactions; Zinc supplementation has been excluded from our analysis because this is not provided in South Africa.

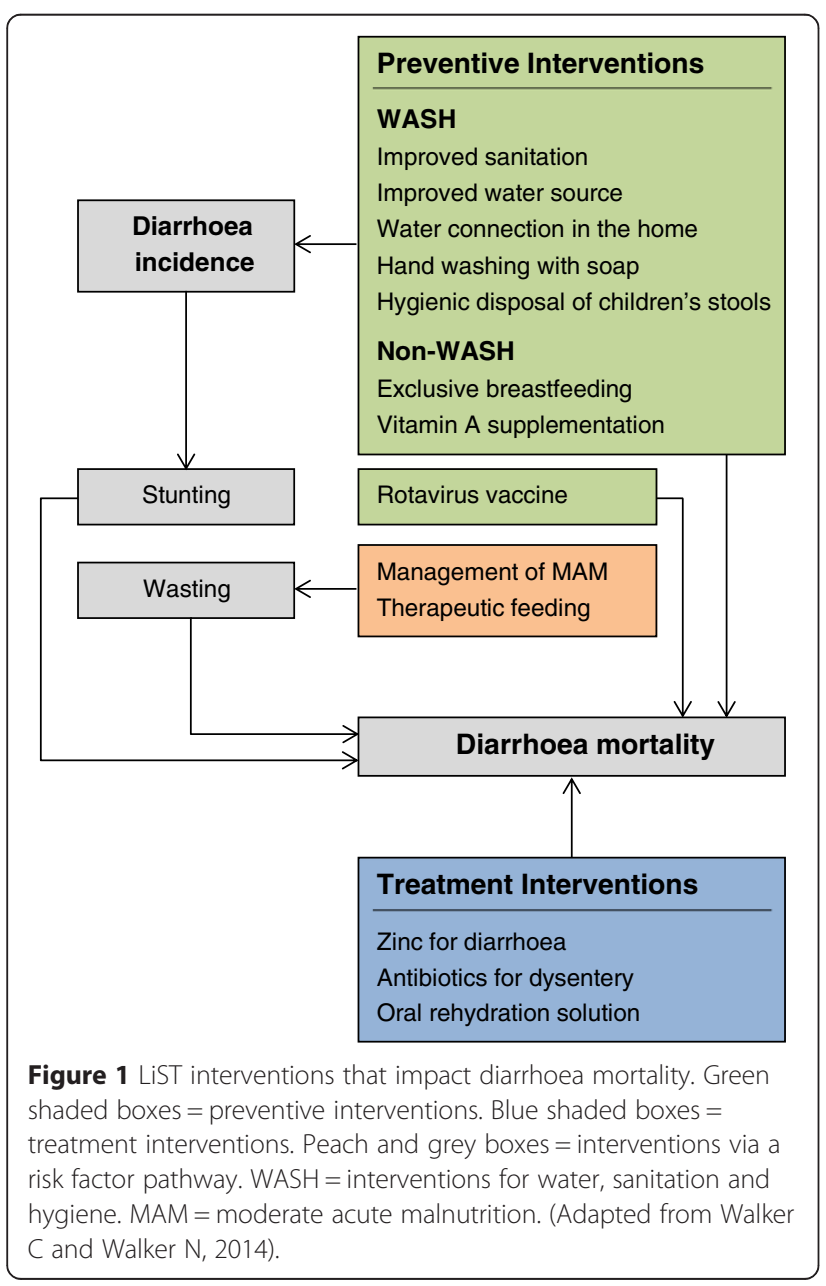

Malnutrition is represented as a risk factor for diarrhoea mortality through the impact of stunting and wasting. Lack of appropriate breastfeeding, vitamin A supplementation and the WASH interventions influence diarrhoea incidence, which in turn affects stunting and subsequent mortality.

The effectiveness values of the diarrhoea interventions included in LiST have been reviewed by the Child Health Epidemiology Reference Group (CHERG) [16-20] and are presented in Additional file 1 [15]. The methods used in our analysis are based on similar multi-country assessments in LiST [21,22].

\section{Scenarios created in LiST}

We assessed the impact of increasing the coverage of 13 interventions on diarrhoeal mortality. The baseline (2014) coverage levels of these interventions were reviewed and modified during a one day expert consultation hosted in South Africa. Twenty-three participants were invited from the health sector, including clinicians, academics and others in positions at national and district level. Coverage levels are indicated in Table 1. Breastfeeding prevalence at baseline was input by age group: $8 \%$ coverage of exclusive breastfeeding for infants younger than 6 months, 51\% coverage of any breastfeeding for infants aged 6-11 months and $31 \%$ coverage of any breastfeeding for infants aged 12 - 24 months [23]. Coverage for the WASH interventions ranged from $17 \%$ for hand washing with soap to $95 \%$ for an improved water source [24]. Unchanged default LiST coverage levels have been indicated. Interventions were linearly scaled up from the baseline year 2014 until 2030, with coverage increases starting in 2015.Three scale up scenarios were implemented: in scenario one, we assumed that the coverage of all interventions increased by $10 \%$ from their baseline estimate (a fixed $0.7 \%$ increase per year); in scenario two, coverage increased by $20 \%$ (a fixed annual increase of $1.3 \%$ ); and in scenario three, coverage for all interventions was increased to $99 \%$ (full coverage) in 2030. In the rest of the document, the scenarios are accordingly referred to as scenario one ( $10 \%$ increase), scenario two (20\% increase) and scenario three (full coverage). Coverage levels for other maternal and child health interventions were not altered, in order to isolate the impact of the priority interventions for diarrhoea prevention and treatment.

The baseline mortality rates used in our analysis were 41 deaths per 1,000 live births for under-five children and 13/1,000 for neonates [3]. The causes of newborn mortality were adapted (South African Medical Research Council: Preliminary estimates for burden of disease in 2010, unpublished) estimates to fit the causal categories in LiST (Figure 2). The categories in LiST differ slightly 
Table 1 Baseline and projected coverage of interventions to prevent and treat diarrhoea

\begin{tabular}{|c|c|c|c|c|}
\hline Intervention & Baseline (2014) & Scenario 1 (+10\%) & Scenario 2 (+20\%) & Scenario 3 (full coverage) \\
\hline \multicolumn{5}{|l|}{ Breastfeeding } \\
\hline Exclusive breastfeeding, $<6$ months & $8[23]$ & 18 & 28 & 99 \\
\hline Any breastfeeding, 6 - 11 months & $51[23]$ & 61 & 71 & 99 \\
\hline Any breastfeeding, 12 - 24 months & $31[23]$ & 41 & 51 & 99 \\
\hline \multicolumn{5}{|l|}{ Feeding and supplements } \\
\hline Vitamin A supplementation & 50 & 60 & 70 & 99 \\
\hline \multicolumn{5}{|l|}{ WASH } \\
\hline Improved water source & $95.1[24]$ & 99 & 99 & 99 \\
\hline Water connection in the home & $79.2[24]$ & 89.2 & 99 & 99 \\
\hline Improved sanitation & $74.4[24]$ & 84.4 & 94.4 & 99 \\
\hline Hand washing with soap & $17^{*}$ & 27 & 37 & 99 \\
\hline Hygienic disposal of children's stools & $40.5^{*}$ & 50.5 & 60.5 & 99 \\
\hline \multicolumn{5}{|l|}{ Vaccines } \\
\hline Rotavirus & $64[9]$ & 74 & 84 & 99 \\
\hline \multicolumn{5}{|l|}{ Diarrhoea treatment } \\
\hline Oral rehydration solution & 50 & 60 & 70 & 99 \\
\hline Antibiotics - for treatment of dysentery & 80 & 90 & 99 & 99 \\
\hline Zinc - for treatment of diarrhoea & 10 & 20 & 30 & 99 \\
\hline \multicolumn{5}{|l|}{ Wasting } \\
\hline Therapeutic feeding - for severe wasting & 45 & 55 & 65 & 99 \\
\hline Treatment for moderate acute malnutrition & 10 & 20 & 30 & 99 \\
\hline
\end{tabular}

*Default coverage level in LiST.

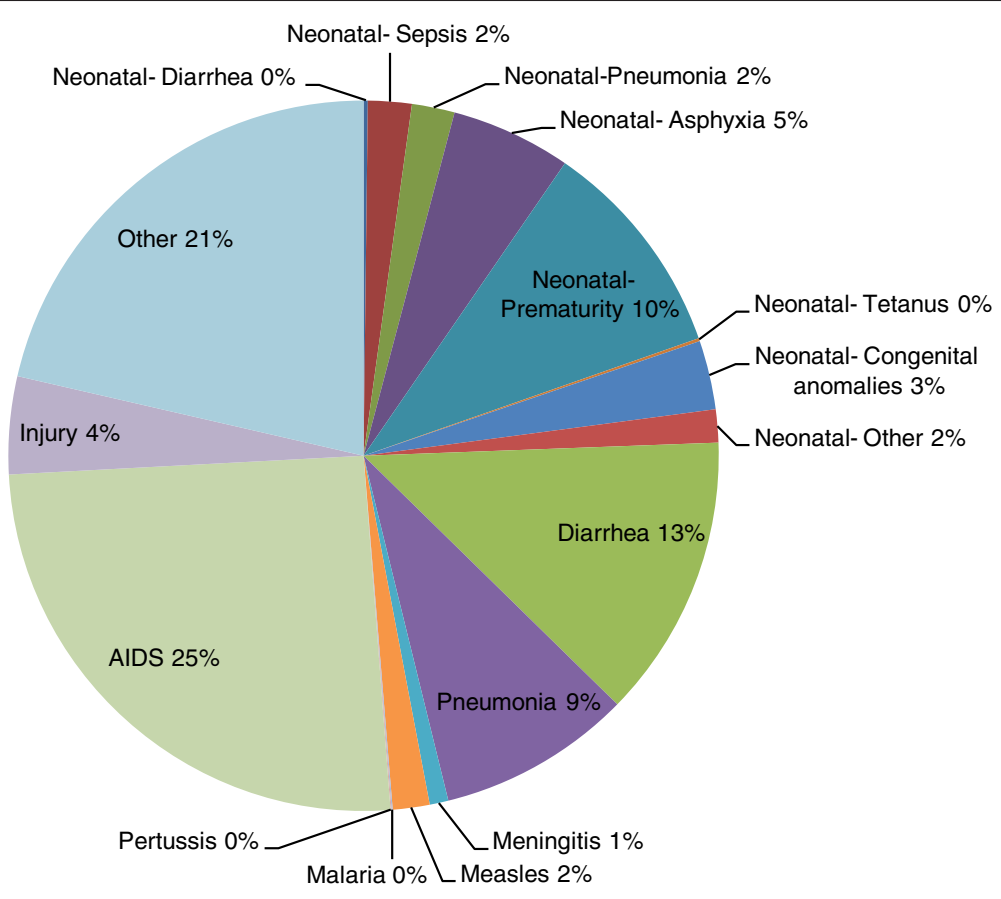

Figure 2 Causes of death in children under-five years, used in LiST (adapted from MRC, 2010). 
from those presented by the MRC. For example, neonatal diarrhoea is not reported separately in the MRC BOD, but rather combined with under-five diarrhoeal deaths. Therefore, we separated these using the default proportions in LiST.

Modelling the cost and impact of diarrhoea interventions Modelling of costs was done using the costing module in LiST, with the most recently available data. The module uses an ingredients approach to costing, based on four components: personnel and labour; drugs and supplies; other recurrent costs; and capital costs. Staff remuneration is based on current salary structures of health workers in South Africa. Salary increases were not applied. The unit costs of drugs and supplies are based on international drug prices from UNICEF and the Management Sciences for Health International Drug Price Indicator $[25,26]$. The unit costs found in LiST were comparable to South African prices of drugs and supplies requested for tender by the Department of Health [27].

The unit costs for WASH programmes are not included in LiST. We estimated these costs using data available from the South African Department of Water and Sanitation [28,29]. A home water connection includes water piped into either the home or yard. This is reflected in the average cost estimate of US\$480 per household (adjusted for inflation). The cost for improved sanitation was estimated using the proportion of South Africans with access to dry and wet sanitation, (60\% and $40 \%$, respectively) [10]. Wet sanitation includes various types of flush latrines and dry sanitation includes pit latrines (with and without ventilation), chemical toilets and bucket toilets. The average household cost for sanitation was approximately US\$900 (adjusted for inflation).

Recurrent costs related to hospitalization and outpatient visits were not included. Recurrent costs include personnel training, gasoline, building rent, office supplies and promotional activities [30,31]. These were outside of the scope of the analysis. In addition, costs estimated in LiST exclude infrastructure development, such as building clinics [30]. All costs were adjusted to 2014 US dollars. Per capita costs use the 2014 South African population estimate of 54 million [32].

Intervention impact was measured in terms of diarrhoeal deaths averted. First, we calculated the expected number of deaths (and cases) at the current (baseline) level of intervention coverage. Second, the number of deaths (and cases) was recalculated for the three intervention scale up scenarios. Deaths averted (or additional lives saved) were then estimated by subtracting the numbers of deaths at baseline from the deaths at increased coverage (the same methodology was used to estimate the number of diarrhoea cases averted).
Ethical review board approval was not required for this analysis as no human subjects were involved.

\section{Results}

Cases averted in 2030

At baseline, the total number of diarrhoea cases was estimated to be 13 million. Intervention scale up would avert approximately 5.3 million in scenario three $(41 \%$ decline), 4.5 million in scenario two (35\% decline) and 3 million diarrhoea cases in scenario one (23\% decline).

\section{Deaths averted in 2030}

Figure 3 illustrates the projected number of deaths due to diarrhoea in each scenario. At baseline (2014), the total number of diarrhoea deaths was estimated to be about 5,500. After scale up of diarrhoea interventions to full coverage (scenario three), the number of deaths would reduce to about 100 in 2030 (98\% decline). This is compared to 1,400 deaths projected in 2030 in scenario two (74\% decline) and 2,800 in scenario one $(48 \%$ decline).

Table 2 shows the additional diarrhoeal deaths prevented by each intervention in the three scenarios in 2030. The percentages in the table indicate the proportion of deaths averted by each intervention for a particular scenario. For example, in scenario three, hand washing with soap averts 1286 diarrhoeal deaths, which constitutes $25 \%$ of the deaths averted. Since water connection in the home is a subset of improved water source, the deaths averted and costs attributed to the two interventions have been combined. The total additional deaths averted were approximately 5,100 in scenario three, 3,700 in scenario two and 2,300 in scenario one.

\section{Intervention costs}

With intervention coverage at $99 \%$ in 2030, total intervention costs were estimated to be US $\$ 2.6$ billion, representing an incremental cost of US\$960 million per year (Table 3). The total costs (incremental costs) were US $\$ 2.5$ billion (US\$830 million) in scenario two and US $\$ 2.2$ billion (US\$510 million) in scenario one. This represents per capita costs of US\$49 for scenario three, US $\$ 47$ for scenario two and US\$41 for scenario one. The incremental costs per capita were US\$18 in scenario three, US\$15 in scenario two and US\$9 in scenario one. The WASH interventions accounted for over $90 \%$ of the total costs in all three scenarios; the two most expensive were improved sanitation and access to safe water. The total costs (incremental costs) of non-WASH interventions were approximately US\$260 million (US\$170 million) in scenario three, US\$150 million (US\$60 million) in scenario two and US\$140 million (US\$40 million) in scenario one. This represents per capita costs of US\$4.9 


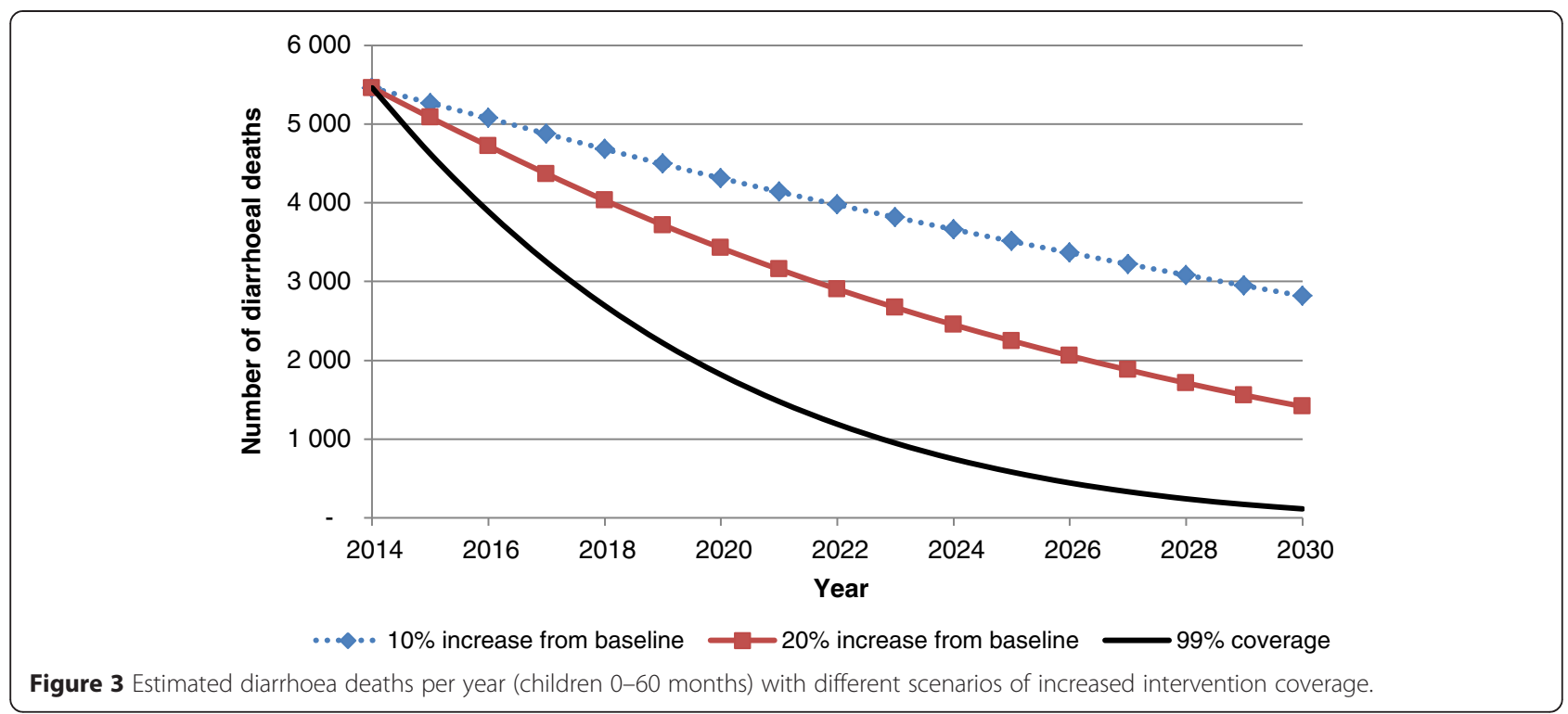

in scenario three, US $\$ 2.8$ in scenario two and US $\$ 2.5$ in scenario one. The incremental costs per capita range from US\$3 in scenario three to less than US\$1 in scenarios one and two.

\section{Discussion}

This analysis determines the impact and costs of interventions for the prevention and treatment of diarrhoea in children under-five in South Africa. LiST was used to model the impact of scaling up 13 essential interventions between 2014 and 2030. Three scenarios were implemented for linear coverage scale up from baseline (2014): coverage increased 10 percentage points by 2030; coverage increased 20 percentage points by 2030; and interventions reached full coverage (99\%) by 2030 .

The results show that scaling up diarrhoeal interventions could contribute significantly to the reduction in child mortality in South Africa. In 2030, diarrhoeal deaths are expected to reduce from an estimated 5,500 in 2014 to 2,800 in scenario one, 1,400 in scenario two and 100 in scenario three. The number of diarrhoea cases is also expected to reduce substantially. Approximately five million cases of diarrhoea can be averted by 2030 if interventions are scaled up to full coverage.

This is the first such analysis and there is no recent South African data with which to compare our results. The GHS conducted by Statistics South Africa in 2010

Table 2 Projected additional diarrhoeal deaths prevented (2030)

\begin{tabular}{|c|c|c|c|}
\hline \multirow[t]{2}{*}{ Interventions } & \multicolumn{3}{|c|}{ Deaths averted } \\
\hline & Scenario 1 & Scenario 2 & Scenario 3 \\
\hline Hand washing with soap & $243(10 \%)$ & $422(11 \%)$ & $1286(25 \%)$ \\
\hline Breastfeeding & $214(9 \%)$ & $370(10 \%)$ & $1034(20 \%)$ \\
\hline Access to safe water* & $656(28 \%)$ & $1100(29 \%)$ & $818(16 \%)$ \\
\hline ORS - oral rehydration solution & $557(24 \%)$ & $704(19 \%)$ & $518(10 \%)$ \\
\hline Hygienic disposal of children's stools & $101(4 \%)$ & $176(5 \%)$ & $382(8 \%)$ \\
\hline Improved sanitation - Utilization of latrines or toilets & $229(10 \%)$ & $488(13 \%)$ & $363(7 \%)$ \\
\hline Vitamin A supplementation & $87(4 \%)$ & $150(4 \%)$ & $286(6 \%)$ \\
\hline Zinc - for treatment of diarrhea & $78(3 \%)$ & $97(3 \%)$ & $130(3 \%)$ \\
\hline Rotavirus & $59(3 \%)$ & $103(3 \%)$ & $135(3 \%)$ \\
\hline Therapeutic feeding - for severe wasting & $57(2 \%)$ & $70(2 \%)$ & $70(1 \%)$ \\
\hline Treatment for moderate acute malnutrition & $9(0.4 \%)$ & $12(0.3 \%)$ & $20(0.4 \%)$ \\
\hline Antibiotics - for treatment of dysentery & $47(2 \%)$ & $56(1 \%)$ & $17(0.3 \%)$ \\
\hline Total & 2337 (100\%) & $3748(100 \%)$ & $5059(100 \%)$ \\
\hline
\end{tabular}

*Access to safe water includes both an improved water source and a water connection in the home. 
Table 3 Projected intervention costs in 2030 (2014 US\$)

\begin{tabular}{|c|c|c|c|c|c|c|}
\hline & \multicolumn{2}{|l|}{ Scenario 1} & \multicolumn{2}{|l|}{ Scenario 2} & \multicolumn{2}{|l|}{ Scenario 3} \\
\hline & Total costs & Incremental costs & Total costs & Incremental costs & Total costs & Incremental costs \\
\hline \multicolumn{7}{|l|}{ WASH } \\
\hline Improved sanitation & $1304343520(59.6 \%)$ & 297114644 (58.4\%) & 1530490650 (60.9\%) & 523262424 (62.7\%) & $1531657562(57.9 \%)$ & $524428690(54.4 \%)$ \\
\hline Access to safe water* & 735212909 (33.6\%) & 163366833 (32.1\%) & 816261686 (32.5\%) & 244415957 (29.3\%) & 816884039 (30.9\%) & 245037965 (25.4\%) \\
\hline Hand washing with soap & $4435223(0.2 \%)$ & $1988942(0.4 \%)$ & $6022585(0.2 \%)$ & $3599383(0.4 \%)$ & $16126764(0.6 \%)$ & 13703561 (1.4\%) \\
\hline Hygienic disposal of children's stools & 8295509 (0.4\%) & $2467605(0.5 \%)$ & $9847740(0.4 \%)$ & $4074817(0.5 \%)$ & $16126764(0.6 \%)$ & 10353840 (1.1\%) \\
\hline Sub total & $2052287161(93.8 \%)$ & 464938024 (91.5\%) & 2362622661 (94\%) & 775352580 (92.9\%) & 2380795129 (90.1\%) & 793524057 (82.3\%) \\
\hline \multicolumn{7}{|l|}{ Non-WASH } \\
\hline Treatment for moderate acute malnutrition & $25051124(1.1 \%)$ & $11841473(2.3 \%)$ & $37300440(1.5 \%)$ & $24205350(2.9 \%)$ & 123459675 (4.7\%) & $110364586(11.4 \%)$ \\
\hline Therapeutic feeding - for severe wasting & 34412469 (1.6\%) & 4719079 (0.9\%) & 40369964 (1.6\%) & 10934337 (1.3\%) & 61670496 (2.3\%) & 32234868 (3.3\%) \\
\hline Breastfeeding & 36424280 (1.7\%) & $26620116(5.2 \%)$ & 36080948 (1.4\%) & 26369276 (3.2\%) & 36081673 (1.4\%) & 26370002 (2.7\%) \\
\hline Zinc - for treatment of diarrhoea & $16296788(0.7 \%)$ & $4045301(0.8 \%)$ & $16378662(0.7 \%)$ & $4236493(0.5 \%)$ & 23874604 (0.9\%) & $11732435(1.2 \%)$ \\
\hline Rotavirus & 16171464 (0.7\%) & $-4090655(-0.8 \%)$ & $12644055(0.5 \%)$ & $-7442129(-0.9 \%)$ & $7898913(0.3 \%)$ & $-12187271(-1.3 \%)$ \\
\hline Vitamin A supplementation & $6205260(0.3 \%)$ & 484905 (0.1\%) & $7018875(0.3 \%)$ & $1318815(0.2 \%)$ & $8272412(0.3 \%)$ & $2572352(0.3 \%)$ \\
\hline Antibiotics - for treatment of dysentery & $657076(0.03 \%)$ & $80262(0.02 \%)$ & $760948(0.03 \%)$ & $189187(0.02 \%)$ & $1079560(0.04 \%)$ & $507799(0.1 \%)$ \\
\hline Oral rehydration solution & $840507(0.04 \%)$ & $-282589(-0.1 \%)$ & $619551(0.02 \%)$ & $-493656(-0.1 \%)$ & $273673(0.01 \%)$ & $-839535(-0.1 \%)$ \\
\hline Sub total & 136058968 (6.2\%) & 43417892 (8.5\%) & $151173443(6 \%)$ & $59317673(7.1 \%)$ & 262611005 (9.9\%) & $170755236(17.7 \%)$ \\
\hline Total costs & 2188346129 (100\%) & 508355915 (100\%) & 2513796104 (100\%) & 834670254 (100\%) & 2643406134 (100\%) & $964279292(100 \%)$ \\
\hline Costs per capita & 41 & 9 & 47 & 15 & 49 & 18 \\
\hline
\end{tabular}

*Access to safe water includes both an improved water source and a water connection in the home. 
estimated that there were approximately 60000 cases of diarrhoea per month in children under-five (about 720 000 per year), and 9,000 diarrhoeal deaths (compared to 5,500 deaths in our model) [2]. The District Health Information System (DHIS), which records data at health facility level, estimated that the under-five incidence of diarrhoea was 90.3 per 1000 in 2012 (approximately 520 000 cases) [33]. Though these data sources provide useful information, they may not be entirely representative. The DHIS records the more severe cases of diarrhoea, since a large number of diarrhoea cases are treated at home and/or by traditional healers [34]. Further, it is worth noting that the landscape in the South African health system has changed significantly since the GHS was undertaken. The rotavirus vaccine was introduced in 2009, fewer babies are born HIV positive, and ARV usage has been scaled up [35]. There has also been investment in infrastructure, contributing to the provision of safe water and sanitation to more households. These factors may account for the lower projected diarrhoeal deaths in the model in 2014. While it is difficult to verify the results of our analysis in the absence of updated burden of disease data, the recent under-five mortality estimate indicates that there has been an overall improvement in the burden of childhood morbidity and mortality, and this likely includes diarrhoea [3].

Preventive interventions are crucial. WASH interventions are shown to avert more than $50 \%$ of the diarrhoeal deaths, but these also amount to more than $90 \%$ of the total intervention costs. Despite many improvements since 1994, South Africa continues to face challenges with implementing home water connections and improved sanitation and there are significant disparities which are not reflected in national statistics. These WASH interventions may thus not be easy to scale up in the timeframe proposed in this analysis. While over $70 \%$ of households in South Africa have access to sanitation and over $90 \%$ have access to an improved water source, an estimated $12.5 \%$ of households in the Eastern Cape province do not have access to any form of sanitation and $14.1 \%$ of households in Kwazulu-Natal province have never had access to water [11]. Considerable effort will be required to ensure these services are delivered to the most marginalised, impoverished and at-risk communities. This will require collaboration with the Department of Health and the Department of Water Affairs, as child mortality and in particular diarrhoeal morbidity and mortality cannot solely be resolved through health systems interventions.

This analysis also shows that breastfeeding could save a large number of child lives if full coverage could be achieved. Exclusively breastfed children are 14 times more likely to survive the first six months of life than those who are not breastfed [36], yet South Africa has low exclusive breastfeeding rates [37]. Community peer counselling has been shown to be highly effective in increasing breastfeeding rates in South Africa [38]. However, there are challenges associated with implementing and maintaining such community-based programmes as they require retention of trained health care workers who are adequately remunerated [39], and there are entrenched community practices that are difficult to overcome [40]. Mothers frequently believe that breast milk is insufficient and they give their infants water, gripe water and non-prescription medicines for general health [40]. This increases the risk of developing diarrhoea from contaminated water and may cause children to become undernourished. When diarrhoea does occur, caregivers most commonly choose to treat their infants at home with ORS [34] and many prepare the solution incorrectly [41]. Addressing these behaviour change issues will require considerable effort, community engagement and resources.

The model isolates interventions that impact diarrhoea by maintaining constant coverage of other child health interventions. However, coverage of the other interventions is likely to increase, resulting in a lower burden of under-five mortality by 2030 . It is therefore possible that we have overestimated the total number of diarrhoea deaths averted.

This national analysis does not take into account the heterogeneity of intervention coverage and diarrhoeal illness across the 52 districts in South Africa. For example, the institutional diarrhoea case fatality rates in the Eastern Cape are 6.9\% compared to the Western Cape at $0.2 \%$ [42]. Furthermore, the paper does not address how increased coverage will be achieved, but the estimated intervention costs can guide policy and budget planning. The additional cost of implementing all 13 interventions will range between US $\$ 508$ million (US\$9 per capita) to US\$964 million (US\$18 per capita) annually. The eight non-WASH interventions would require an additional investment ranging from US $\$ 43$ million (less than US\$1 per capita) to US $\$ 170$ million (US\$3 per capita) per year. These costs are within South Africa's allocated health budget (approximately US\$14.7 billion in 2014/15) [43].

The cost projections are likely an underestimate as staff salary increases were not taken into account and infrastructure development is not fully considered. For the WASH interventions, household infrastructure has been estimated, yet broader system infrastructure requirements, such as waste management systems, have not been considered. Furthermore, the model assumes that the health system interventions are delivered at uniformly high quality. This is unlikely given drug shortages, health care worker attitudes and institutional challenges. Significantly more resources are probably required to address such issues. 
HIV/AIDS is a major burden in South Africa. In 2008, approximately $3 \%$ of children $0-4$ years of age were HIV-positive [44], and this increases the risk of diarrhoeal mortality 11 times [5]. The model does not however explicitly incorporate the relationship between HIV/AIDS and diarrhoea. This should be included in future analyses.

The long term consequences of diarrhoeal illness have not been incorporated in this analysis. Early childhood diarrhoea is associated with impaired physical and cognitive development. The resulting losses in human potential and economic productivity may have a greater impact than the burden of diarrhoeal mortality [45]. Furthermore, the full impact of the 13 interventions, on conditions other than diarrhoea, has not been explored. Increased breastfeeding, for example, is associated with a reduction in several childhood illnesses including respiratory infections, gastroenteritis, otitis media and necrotising enterocolitis [46]. Similarly, other interventions could have benefits in addition to reducing diarrhoea morbidity and mortality. WASH interventions will address neglected tropical diseases which account for significant disabilityadjusted life years (DALYs) in low and middle income countries [47], and have significant impacts not only on health, but also on social and economic development [48]. The impact and costs of these additional benefits should be taken into account in future research.

\section{Conclusion}

Diarrhoea is responsible for a substantial number of child deaths in South Africa [2], and reducing its impact could help South Africa attain its post MDG child health targets. This analysis shows that scaling up 13 interventions to full coverage (99\%), could prevent more than 5 million diarrhoea cases and 5000 diarrhoeal deaths in children under-five. Progress has been made by introducing the rotavirus vaccine, adopting the WHO guidelines on infant feeding and attaining the water and sanitation targets of MDG 7. However, there is still a need for increased coverage of exclusive breastfeeding and improved home water and sanitation infrastructure. Given South Africa's health budget, the cost of scaling up the 13 interventions should be affordable, with the estimated additional costs for the non-WASH health system interventions ranging between under US $\$ 1$ and US $\$ 3$ per capita.

\section{Additional file}

Additional file 1: Parameters used in the Lives Saved Tool that impact diarrhoea. Source: Fischer Walker CL, Walker N. The Lives Saved Tool (LiST) as a model for diarrhea mortality reduction. BMC Med. 2014;12:70.

\section{Competing interests}

The authors declare that they have no competing interests.

\section{Authors' contributors}

All authors were responsible for the study concept, design, and interpretation of data. LC and JM collected the data, performed the analysis and drafted the manuscript. AT and $\mathrm{KH}$ reviewed and provided comments on the manuscript. All authors read and approved the final manuscript.

\section{Acknowledgements}

PRICELESS SA is funded by the South Africa Medical Research Council; grant fund number D1305910-01.

Received: 4 November 2014 Accepted: 26 March 2015

Published online: 17 April 2015

\section{References}

1. Liu L, Johnson HL, Cousens S, Perin J, Scott S, Lawn JE, et al. Global, regional, and national causes of child mortality: an updated systematic analysis for 2010 with time trends since 2000. Lancet. 2012;379(9832):2151-61.

2. Statistics South Africa: Levels and trends of morbidity and mortality among children aged under-five years in South Africa, 2006-2010. Pretoria, South Africa: Statistics South Africa; 2012.

3. Dorrington RE, Bradshaw D, Laubscher R. Rapid mortality surveillance report 2012. Cape Town, South Africa: South African Medical Research Council; 2014.

4. UNICEF: Pneumonia and diarrhoea: tackling the deadliest diseases for the world's poorest children. UNICEF; 2012

5. Tindyebwa D, Kayita J, Musoke P, Eley B, Nduati R, Coovadia H, et al. Handbook on paediatric AIDS in Africa by the African Network for the Care of Children Affected by AIDS: USAID; 2006.

6. Bamford L. Maternal, Newborn and Child Health. In: Padarath A, English R, editors. South African Health Review. Durban: Health Systems Trust; 2013.

7. UNICEF, WHO: Diarrhoea: Why children are still dying and what can be done? WHO Bulletin; 2009

8. Madhi SA, Cunliffe NA, Steele D, Witte D, Kirsten M, Louw C, et al. Effect of Human Rotavirus Vaccine on Severe Diarrhea in African Infants. N Engl J Med. 2010;362(4):289-98.

9. UNICEF, WHO. Immunisation summary: A statistical reference containing data through 2013. WHO; 2014

10. Statistics South Africa: Water and sanitation 2002-2010: in-depth analysis of the General Household Survey data. Statistics South Africa; 2011

11. South African Human Rights Commission: Report on the right to access sufficient water and decent sanitation in South Africa. South African Human Rights Commission; 2014

12. Stover J, McKinnon R, Winfrey B. Spectrum: a model platform for linking maternal and child survival interventions with AIDS, family planning and demographic projections. Int J Epidemiol. 2010;39 Suppl 1:i7-10.

13. Walker N, Tam Y, Friberg IK. Overview of the Lives Saved Tool (LiST). BMC Public Health. 2013;13 Suppl 3:S1.

14. LiST: The Lives Saved Tool. An evidence-based tool for estimating intervention impact [http://www.jhsph.edu/dept/ih/IIP/list/index.html]

15. Fischer Walker CL, Walker N. The Lives Saved Tool (LiST) as a model for diarrhea mortality reduction. BMC Med. 2014;12:70.

16. Cairncross S, Hunt C, Boisson S, Bostoen K, Curtis V, Fung IC, et al. Water, sanitation and hygiene for the prevention of diarrhoea. Int J Epidemiol. 2010;39 Suppl 1:1193-205.

17. Walker $\mathrm{CL}$, Black RE. Zinc for the treatment of diarrhoea: effect on diarrhoea morbidity, mortality and incidence of future episodes. Int J Epidemiol. 2010;39 Suppl 1:i63-69.

18. Munos MK, Walker CL, Black RE. The effect of oral rehydration solution and recommended home fluids on diarrhoea mortality. Int J Epidemiol. 2010;39 Suppl 1:i75-87.

19. Munos MK, Walker $C L$, Black RE. The effect of rotavirus vaccine on diarrhoea mortality. Int J Epidemiol. 2010;39 Suppl 1:i56-62.

20. Traa BS, Walker CL, Munos M, Black RE. Antibiotics for the treatment of dysentery in children. Int J Epidemiol. 2010;39 Suppl 1:i70-74.

21. Fischer Walker CL, Friberg IK, Binkin N, Young M, Walker N, Fontaine O, et al. Scaling up diarrhea prevention and treatment interventions: a Lives Saved Tool analysis. PLoS Med. 2011;8(3), e1000428.

22. Bhutta ZA, Das JK, Walker N, Rizvi A, Campbell H, Rudan I, et al. Interventions to address deaths from childhood pneumonia and diarrhoea equitably: what works and at what cost? The Lancet. 2013;381(9875):1417-29.

23. UNICEF. Every Child Counts: The State of the World's Children. UNICEF; 2014 
24. UNICEF, WHO: Joint Monitoring Programme for Water Supply and Sanitation. Progress on drinking water and sanitation 2014.

25. UNICEF Supply Catalogue [www.supply.unicef.org]

26. Management Sciences for Health, Word Health Organization: International drug price indicator guide. Frye JE (Eds). Cambridge, MA: WHO; 2012

27. Tender section [http://www.health.gov.za/tenders.php]

28. Department of Water Affairs and Forestry: Guideline for the costing of household sanitation projects: DWAF; 2007.

29. City of Johannesburg: Amendment of tariff of charges for water services, Annexure 21: City of Johannesburg; 2012.

30. Bhutta ZA, Das JK, Bahl R, Lawn JE, Salam RA, Paul VK, et al. Can available interventions end preventable deaths in mothers, newborn babies, and stillbirths, and at what cost? The Lancet. 2014;384(9940):347-70.

31. Stenberg $K$, Axelson $H$, Sheehan $P$, Anderson I, Gülmezoglu AM, Temmerman $\mathrm{M}$, et al. Advancing social and economic development by investing in women's and children's health: a new Global Investment Framework. Lancet. 2014;383(9925):1333-54.

32. Statistics South Africa: Mid-year population estimates 2014. Statistics South Africa; 2014.

33. District Health Information Systems Database [http://indicators.hst.org.za/ healthstats/132/data]

34. Friend-du Preez N, Cameron N, Griffiths P. The importance of medicines in health-seeking behaviour for childhood illnesses in urban South Africa. Soc Sci Med. 2013:92:43-52.

35. Venter F: HIV Treatment in South Africa: The challenges of an increasingly successful antiretroviral programme. South African Health Review 2012/13 2013

36. Chidiebere OD, Stanley O, Joy E, Clement E, Ifeyinwa N, Uchenna E: Effect of Exclusive Breastfeeding on Incidences of Illness in Infant's First 6 Months of Life. Journal of Pediatrics and Neonatal Care 2014, 1(4): 00025. doi: 10.15406/jpnc.2014.01.00025.

37. Tylleskar T, Jackson D, Meda N, Engebretsen IM, Chopra M, Diallo AH, et al. Exclusive breastfeeding promotion by peer counsellors in sub-Saharan Africa (PROMISE-EBF): a cluster-randomised trial. Lancet. 2011;378(9789):420-7.

38. Bland RM, Little KE, Coovadia HM, Coutsoudis A, Rollins NC, Newell ML. Intervention to promote exclusive breast-feeding for the first 6 months of life in a high HIV prevalence area. AIDS (London, England). 2008;22(7):883-91.

39. Nkonki L, Cliff J, Sanders D. Lay health worker attrition: important but often ignored. Bull World Health Organ. 2011;89(12):919-23.

40. Goosen C, MCLachlan M, Schubl C. Factors impeding exclusive breastfeeding in a low-income area of the Western Province of South Africa. Africa Journal of Nursing and Midwifery. 2011;16(1):13-31.

41. Mawela MP, de Villiers FP. The effect of admission on oral rehydration-related knowledge. Ann Trop Paediatr. 1999;19(1):75-81.

42. Massyn N DC, Peer N, Padarath A, Barron P, English R, : District Health Barometer 2013/14. Health Systems Trust 2014

43. National Budget [http://www.treasury.gov.za/documents/national\% 20budget/2014/]

44. Shisana O, Simbayi L, Rehle T, Zungu N, Suma K, Ngogo N, et al. South African National HIV Prevalence, Incidence, Behaviour and Communication Survey, 2008: The Health of our Children. Cape Town: HSRC Press; 2010.

45. Guerrant RL, Kosek M, Moore S, Lorntz B, Brantley R, Lima AAM. Magnitude and Impact of Diarrheal Diseases. Arch Med Res. 2002;33(4):351-5.

46. Kramer M, Kakuma R. The optimal duration of exclusive breastfeeding Cochrane Database of Systematic Reviews 2002, Issue 1. Art. No.: CD003517. DOl: 10.1002/14651858.CD003517

47. Hotez PJ, Molyneux DH, Fenwick A, Kumaresan J, Sachs SE, Sachs JD, et al. Control of neglected tropical diseases. N Engl J Med. 2007;357(10):1018-27.

48. Mara D, Lane J, Scott B, Trouba D. Sanitation and Health. PLoS Med. 2010;7(11), e1000363.

\section{Submit your next manuscript to BioMed Central and take full advantage of:}

- Convenient online submission

- Thorough peer review

- No space constraints or color figure charges

- Immediate publication on acceptance

- Inclusion in PubMed, CAS, Scopus and Google Scholar

- Research which is freely available for redistribution

Submit your manuscript at www.biomedcentral.com/submit 